\title{
Irrigation System Designed with SCADA and Wireless Sensor Network Applied to the Colombian Environment
}

\author{
Camilo Jiménez M, Julián R. Camargo L and César A. Perdomo Ch* \\ Universidad Distrital Francisco José de Caldas, Bogotá D.C., Colombia; \\ acjimenezm@correo.udistrital.edu.co, jcamargo@udistrital.edu.co, \\ cperdomo@udistrital.edu.co
}

\begin{abstract}
Objectives: This document describes an automatic irrigation system design, using SCADA software and wireless sensor network. Methods/Analysis: The system was made using the drip irrigation system method, and the communication the field and the supervisor system was established using ZigBee devices connected in mesh topology. The SCADA system monitors four variables of the field: temperature, soil moisture content, water and nutrient level and status of the ZigBee device. Findings: The purpose of this paper is not only to contribute knowledge to the development of the Colombian field, through the application of new technologies of irrigation automation of crops, but to motivate research, development and innovation around this topic. Improvements: It is contemplated the use of a web portal where you can monitor the system, thus adding a notification mode. In addition, the use of redundancy to avoid loss of data in the crop, both on the side of data acquisition, and on the side of storage.
\end{abstract}

Keywords: Data Logging, Drip Irrigation, Precision Agriculture, Supervision-Control And Data Acquisition (SCADA), ZigBee

\section{Introduction}

The development of the agricultural sector is key to the future of a nation. Specifically for the Colombian case, there is a potential for growth that has been wasted, and although its contribution to the Gross Domestic Product (GDP) has decreased ${ }^{1}$, it is one of the essential activities in the economic growth that allows the development of rural areas (especially in developing countries), employment generation, poverty reduction, food security and improvement of quality of life in the countryside.

Agriculture is a high importance sector and it is estimated that the demand for food will grow considerably in the future, approximately $70 \%$ due to the growth of the world population to 9 billion in $2050^{2}$, which is why the sector is required Colombian agricultural is competitive.
To achieve this, new information technologies play an important role in improving efficiency and costs in a process ${ }^{3}$. Therefore, its implementation in the agricultural sector should be considered. Obtaining the status of the crop is important to be able to manage it properly. For example, to know the amount of fertilizer and nutrients that will be need it, to prevent the arrival of pests, to maintain the quality of the product, among others ${ }^{4}$. In some cases, this is done manually by the farmer, a task that costs time and money.

To overcome this problem, it have been chosen make use of new technologies that help manage the crop using different data sources, and consequently save time and money. This is called Precision Agriculture, and it is a trend that is revolutionizing the agricultural process in the world ${ }^{5}$. It covers practices related to crop management.

${ }^{*}$ Author for correspondence 
One of the relevant applications is the management of water and nutrients using irrigation methods. Due to climate change and water scarcity (increasingly notorious), attention is focused on irrigation technologies that use little water and energy ${ }^{6}$.

The requirements to automate agricultural processes are increasing, and focus on making economic and efficient systems to control different variables, using little electricity and water ${ }^{7}$, which leads to the reduction of waste generated, all this finally to be competitive in the market.

Among the existing irrigation methods, the drip system makes efficient use of water and nutrients ${ }^{7}$. The water is distributed slowly and uniformly to the roots of the plants through narrow tubes and valves, installed throughout the crop. However, irrigation does not guarantee on its own that there is no waste of water and nutrients. That is why there must be an additional process that allows determining the precise amount of water and nutrients that the crop needs, as well as the period of time in which they must be irrigated. For this, the crop is required to provide information on the current state of the variables that affect it directly.

Recently, wireless sensor networks have emerged as a promising technology in the field of embedded systems ${ }^{-}$. The potential of these networks depends on the integration of different sensors, from the simplest (temperature, humidity, pressure) to the most complex (GPS, image processing, radar) ${ }^{9}$. The latest work has focused on applications with low power consumption, low data rate, and increasingly smaller devices. The use of sensor networks in agriculture, specifically in irrigation systems, could optimize the consumption of water and nutrients, through remote access to crop variables, contributing to a reduction in costs and a lower environmental impact ${ }^{10}$.

From the practical point of view, the automation should allow an easy management of the parameters of the plant, to reduce the resistance of the farmer to the use of new technologies. In this context, a supervision system (Supervision-Control And Data Acquisition (SCADA)-HMI) is essential to create the interface between farmers and field teams. SCADA systems used for process automation show a growth in their use in recent years ${ }^{11}$, covering almost all types of industries, such as automotive, oil, aqueducts, food and beverages, gas transportation lines, among others. The common factor in these industries is that it requires monitoring and control of variables of the process at a distance, similar to an agricultural process.

Using a monitoring system, the farmer, in addition to observing the real-time status of crop variables, can store pertinent information to keep a historical record of their behavior, and in turn generate periodic reports of it. In addition, an alarm recorder could be configured to inform the farmer in a timely manner about crop anomalies, by sending email when an alarm is activated $12-16$.

\section{General Description of the Designed System}

Figure 1 shows the general diagram of the system, together with its components. Each component is described in the following sections.

It is decided to use the drip irrigation method, since it allows obtaining maximum results in production harvest

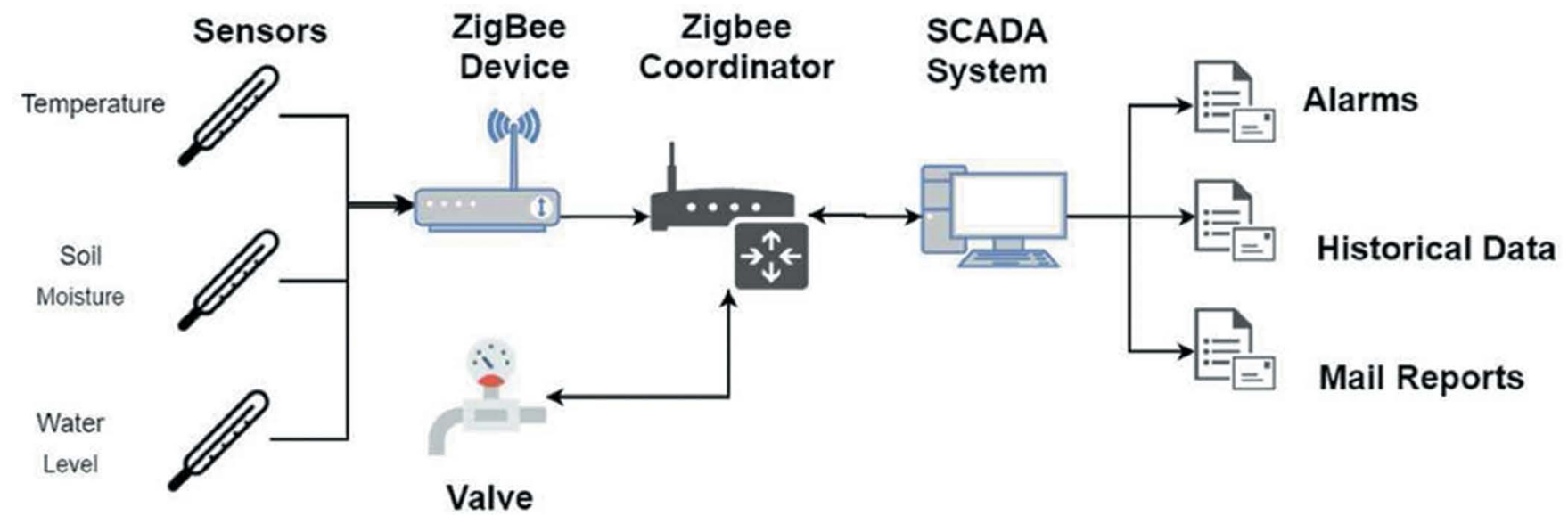

Figure 1. General diagram of the proposed system. 
quality while minimizing the use of water, energy and other resources.

\section{Selection of Components of the Proposed System}

\subsection{Criteria for the Selection of System Components}

The sensors to be used in the design of the wireless sensor network will be of soil temperature and humidity only, so the following criteria should be taken into account:

Type: They can be active or passive: Passive sensors are low energy devices. Active sensors include radar and sonar is high power consumption systems.

Power consumption: In many applications, the sensors are deployed in remote areas, so the life of a node can be determined by the battery life, so a minimization of energy consumption is necessary.

Operating environment: It is important to know the environment where the sensors will be deployed, since environmental conditions can affect the performance of the sensors.

Communication: The wireless network generally has a limited bandwidth; Networks can be forced to use a noisy channel; and the communication channel can be relegated to an unprotected frequency band ${ }^{17}$. The implications are limited reliability, poor quality of service and security exposure.

Connectivity, telemetry and control of remote devices: The sensors must have the ability to connect to other devices, so they can be monitored remotely and thus perform automatic control processes on a system.

\subsection{Analysis of the Sensors}

\subsubsection{Temperature}

Table 1 show the characteristics of most of the sensors available in the market, which can be used to make comparisons between them, and select the appropriate ones for the development of the design.

For design purposes it is assumed that the ambient temperature of the irrigation zone is between $5^{\circ} \mathrm{C}$ and $30^{\circ} \mathrm{C}$, based on this premise, the appropriate temperature sensor can be selected for the project. The thermocouple and RTD are better in requirements for stability, accuracy and response speed, than in the thermistor and the temperature transducer. However, their costs are high, compared to the other two.

Reviewing the characteristics of the thermistor, it can be inferred that of the four sensors it offers the least benefits, because its linearity and stability is not good and it is highly sensitive. This could explain its low cost. Unlike the thermistor, the criteria of the temperature transducer are good, except for the high sensitivity that it presents.

Due to the above, it was decided that RTD is the sensor that best fits the requirements of the project, despite its cost.

\subsubsection{Soil Moisture}

Table 2 shows the characteristics of four types of commercial sensors, used to measure soil moisture.

According to Table 2, the neutron probe is very expensive and difficult to operate, due to its size. Gypsum blocks, despite their low cost and low sensitivity, are not stable and have little linearity, which makes them the last option, when compared with other soil moisture sensors. Finally, the FDR and TDR probes are compared, which are the ones that best meet the requirements of this work.

Table 1. Criteria for selecting temperature sensors in an irrigation field

\begin{tabular}{lllll}
\hline Criterion & Thermocouple & RTD & Thermistor & $\begin{array}{l}\text { Integrated circuit } \\
\text { temperature transducer }\end{array}$ \\
\hline Rank & $-200^{\circ} \mathrm{C}$ to $1372^{\circ} \mathrm{C}$ & $-50^{\circ} \mathrm{C}$ to $400^{\circ} \mathrm{C}$ & $-55^{\circ} \mathrm{C}$ to $125^{\circ} \mathrm{C}$ & $-40^{\circ} \mathrm{C}$ to $260^{\circ} \mathrm{C}$ \\
Stability & Good & Excellent & Regular & Good \\
Precision & Half & High & Half & Half \\
Repetitiveness & Regular & Excellent & Good & Excellent \\
Sensitivity & Low & Half & Very high & high \\
Response speed & Quick & Half & Half & Quick \\
Linearity & Regular & Good & Deficient & Excellent \\
Size & Big & Medium & Medium & Little \\
Cost & High & High & Low & Low \\
\hline
\end{tabular}


However, the cost, stability and size of the FDR probes make it the type of humidity sensor selected for this job.

\subsection{Analysis of Communication Protocols}

In the development of this work, different communication protocols were found that are used in wireless sensor networks. Next in Table 3 a comparative analysis between them is carried out. According to the comparison made between the found technologies, it is determined that ZigBee is the appropriate protocol for the development of this work, for the following reasons:

- It is the one with the lowest energy consumption, approximately $30 \mathrm{~mA}$ maximum in operational mode, and even less when it is in inactive mode. The autonomy of a ZigBee device can reach 3 years,
- The operating frequency band of ZigBee is free, so it is not necessary to acquire a license to use it,

- It has a range between 10 and 100 meters, but can be extended up to 1000 meters, using gateways, routers and more nodes,

- The implementation of a ZigBee network is less expensive and easier, than if it were done with any of the other protocols,

- ZigBee allows you to reach remote areas where the electric power grid is non-existent,

- The main use of ZigBee devices is for monitoring and data monitoring, which is adjusted for later connection to a SCADA system, purpose of this work,

- Although its bit rate is the lowest of all the protocols analyzed, the most important thing is the fidelity

Table 2. Criteria for selecting soil moisture sensors in irrigation field

\begin{tabular}{lllll}
\hline Criterion & FDR probes & TDR sensor & Gypsum blocks & Neutron probe \\
\hline Stability & Good & Regular & Regular & Excellent \\
Precision & high & high & Half & High \\
Repetitiveness & Excellent & Excellent & Regular & Excellent \\
Sensitivity & high & high & Low & High \\
Response speed & Quick & Quick & Slow & Quick \\
Linearity & Excellent & Excellent & Regular & Good \\
Size & Little & Medium & Medium & Big \\
Cost & Very low & High & Low & Very high \\
\hline
\end{tabular}

Table 3. Comparison of protocols used in wireless sensor networks

\begin{tabular}{|c|c|c|c|c|}
\hline & $\operatorname{ZigBee}^{17-\underline{18}}$ & $\mathbf{W i}-\mathbf{F i} \mathbf{i}^{19}$ & Bluetooth $^{\underline{20-21}}$ & $3 \mathrm{G}-2.5 \mathrm{G}^{22}$ \\
\hline Type of network & WSN & WLAN & PAN & WAN/MAN \\
\hline Application focus & Monitoring and control & Data and voice-IP & Cable replacement & $\begin{array}{l}\text { Broad area of voice and } \\
\text { data }\end{array}$ \\
\hline Bandwidth & 20 kbps - 250 kbps & 2-54 Mbps & 1-3 Mbps & 64 kbps - 128 kbps \\
\hline Transmission range $(\mathrm{m})$ & $10-100$ & $50-100$ & $10-100$ & $>900$ \\
\hline Energy consumption & $30 \mathrm{~mA}$ & $100 \mathrm{~mA}$ & $30 \mathrm{~mA}$ & $100 \mathrm{~mA}$ \\
\hline Size & Smaller & Big & Little & Medium \\
\hline $\begin{array}{l}\text { Max. Number of nodes } \\
\text { per master }\end{array}$ & 100 & 32 & 7 & NA \\
\hline Characteristics & $\begin{array}{l}\text { Reliability, low energy } \\
\text { use and cost }\end{array}$ & $\begin{array}{l}\text { Support, scalability } \\
\text { and cost }\end{array}$ & Cost, ease of use & $\begin{array}{l}\text { Transmission quality } \\
\text { and range }\end{array}$ \\
\hline
\end{tabular}


of the information received, one of the ZigBee features, and

- It is the protocol most used in the use of wireless sensor networks, especially in agriculture.

\subsection{Selection of the ZigBee Network Topology}

To select the best topology for the wireless network with ZigBee devices, the simulation of three topologies (star, mesh and tree) was carried out for 10 hours, in order to determine the one with the highest performance, efficiency and lowest traffic loss. For this, an irrigation area of $500 \mathrm{~m} \times 500 \mathrm{~m}$ and 10 nodes were assumed between coordinators, final devices and routers.

The Riverbed Modeler Academic Edition software was used to perform the simulation. It is used to design and simulate networks with different communication protocols. The advantage of using this software is its easy installation and use in addition, it gives the user information relevant to the performance of the network in a certain time of simulation, such as the rate of sending/ receiving data, delays and lost packets, among others. However, because it is paid software, only the academic version was used, which is limited in some functions and only allows a maximum simulation time of ten (10) hours, but for the purposes of this work it's enough.

Figure 2-4 show the ZigBee topologies to be simulated in order: star, mesh and tree.

The performance graph shown in Figure 5 shows that the mesh topology (blue) has better performance indicators than tree and star topologies, since it has an average transfer rate of 25.800 bits per second. On the contrary, the star topology is the worst performance, with an average of 23.600 bits per second.

According to the delay graph shown in Figure 6, it is observed that the star topology is the one with the most delay between the three, with an average value between 0.0071 and 0.0072 seconds, while the mesh topology is the one less delay time presents, with values between 0.0066 and 0.0067 seconds.

According to Figure 7, the mesh topology is the one with the highest amount of traffic, with an average of 29.200 bits per second, followed very closely by the tree topology, whose sending rate is approximately 28.900 bits. However, the least amount of traffic is sent using the star topology, which reaches 26.800 bits per second.
Analyzing the graphs obtained from the simulation, it can be inferred that the least efficient topology is star topology, because it is the one with the lowest performance, the one with the longest delay time and the one with the lowest data rate (sent/received). On the other hand, mesh topology has the best results, so it can also be inferred that the fact of adding routers allows obtaining better results than when they are not present.

\section{System of Supervision, Control, and Data Acquisition}

For the monitoring, control and data acquisition system, Wonderware was used, which is a suite of components that allow monitoring the real-time and historical behavior of a system. Wonderware is characterized by its ability to work with different industrial communication protocols such as Modbus, Profibus, ABCIP, MELSEC, among others.

The monitoring system was carried out with the objective of monitoring the variables delivered by each ZigBee device, which in this case correspond to temperature, soil moisture, sensor status, and water and nutrient levels. It consists of the following functionalities:

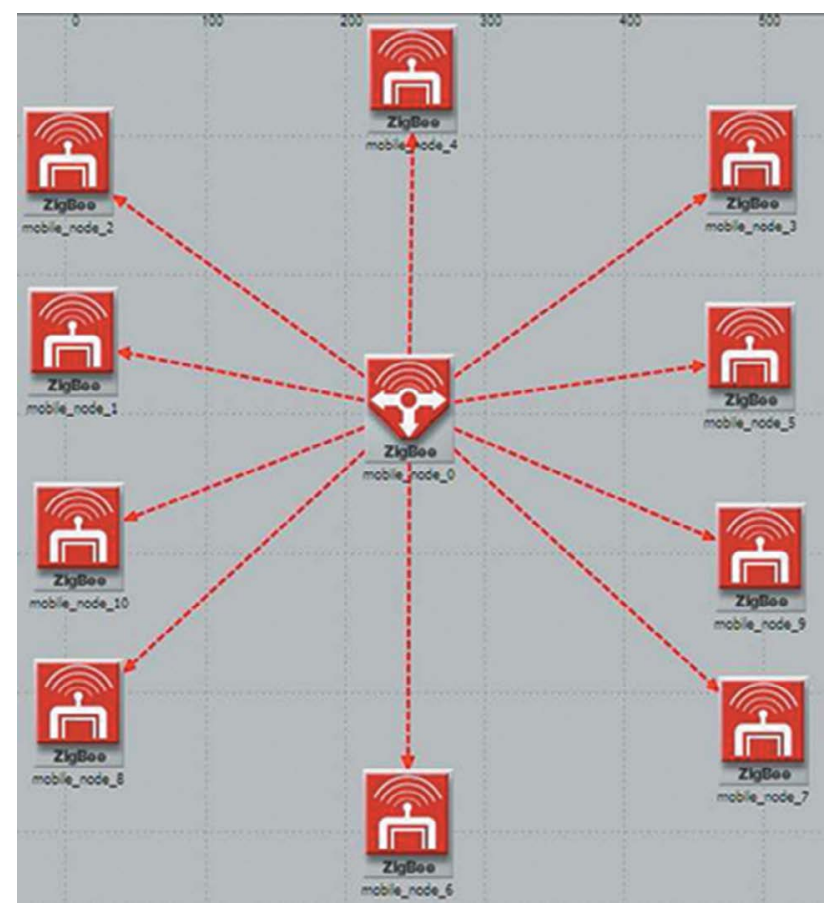

Figure 2. Simulation Star topology with 10 ends nodes and a coordinator node. 


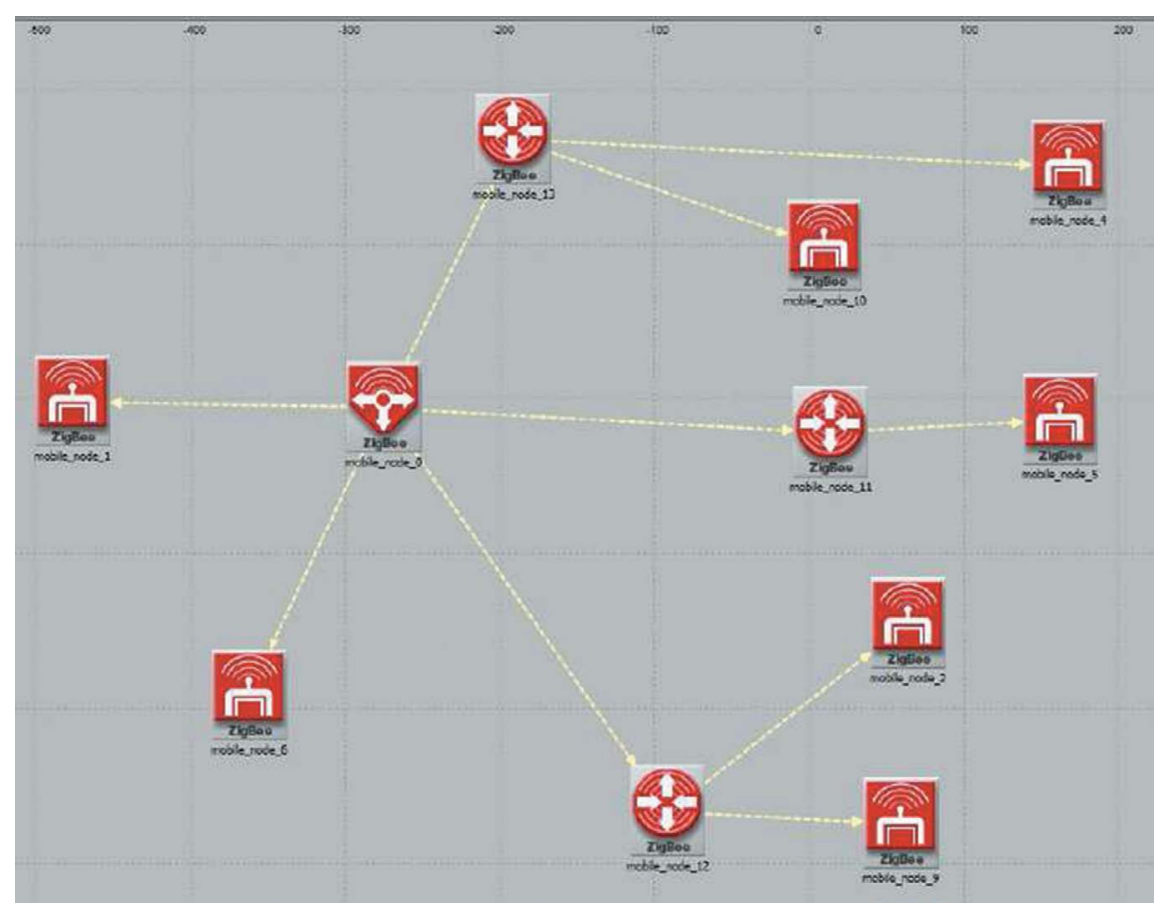

Figure 3. Simulation mesh topology with 7 end nodes, 3 routers and a coordinator node.

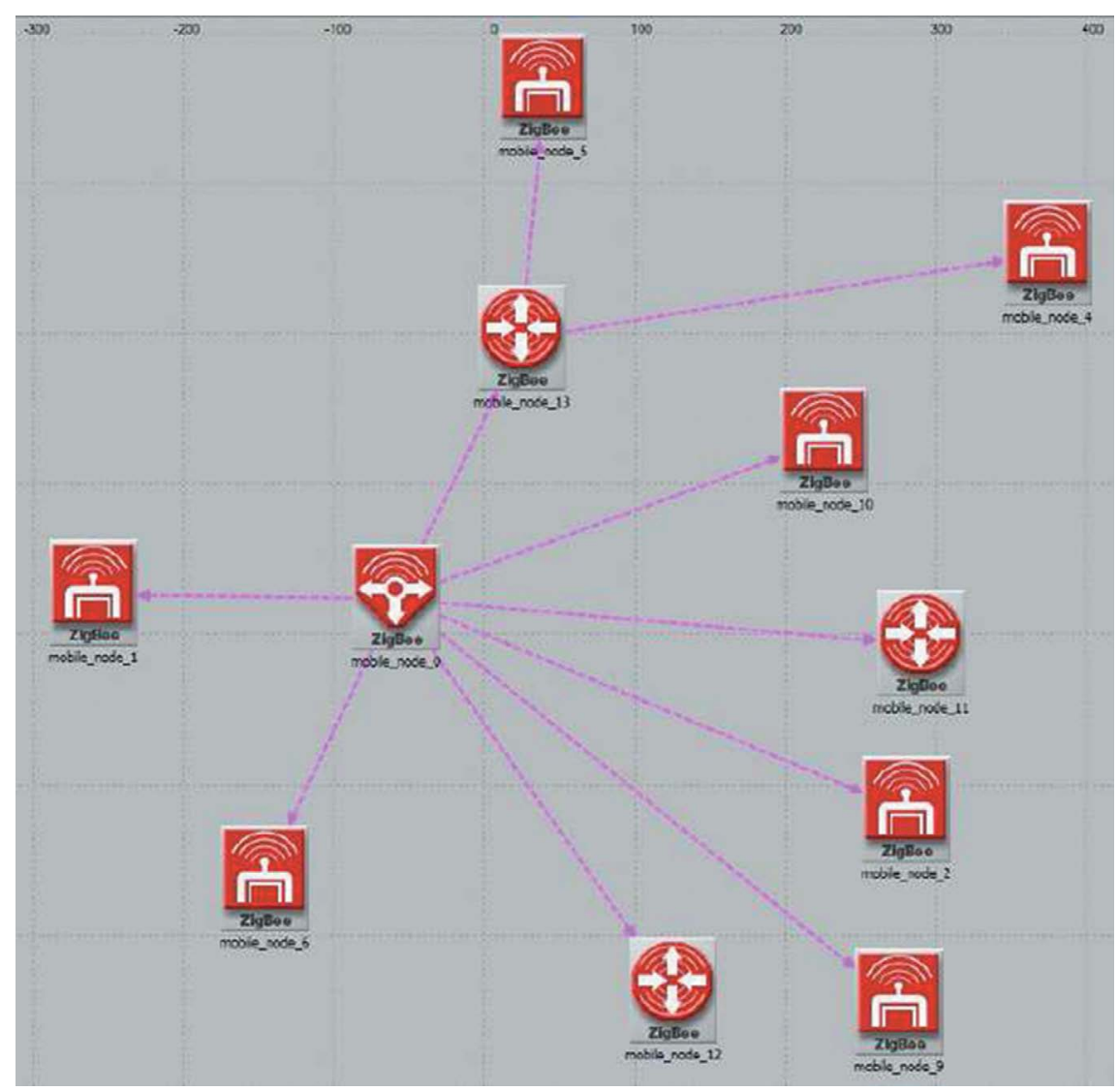

Figure 4. Simulation Tree topology with 7 end nodes, 3 routers and a coordinator node 


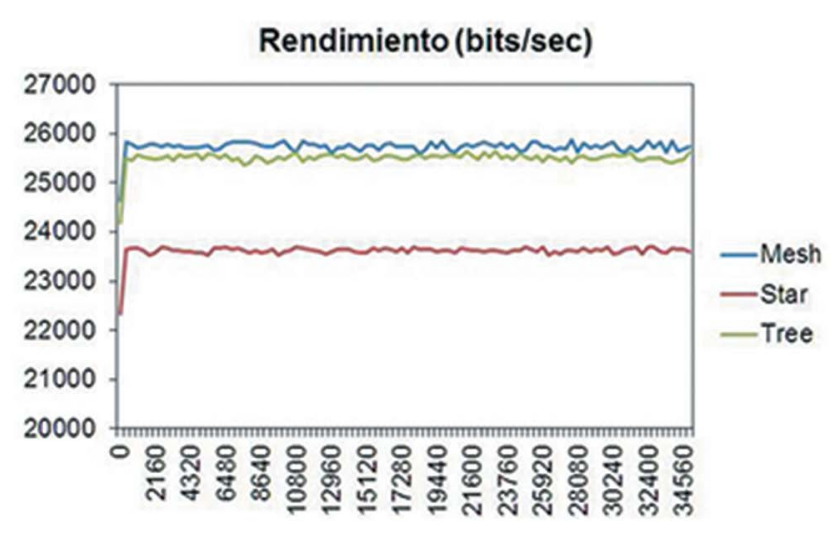

Figure 5. Rate of performance of each of the topologies in 10 hours of simulation.

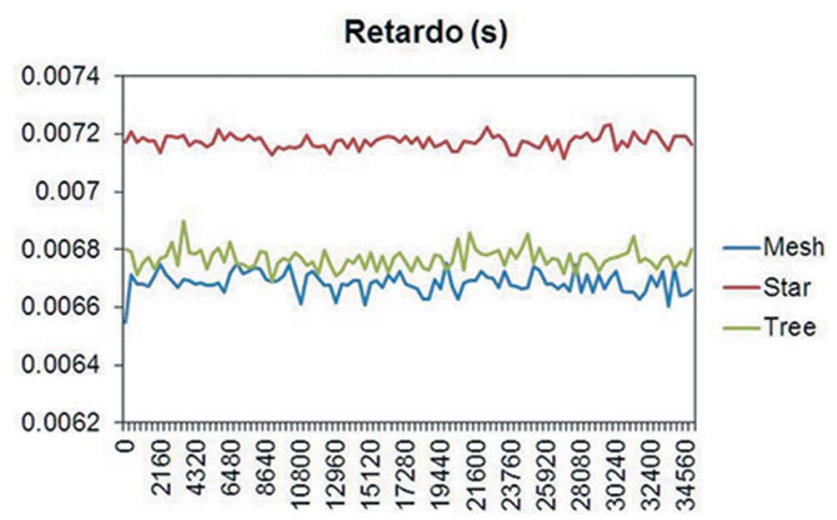

Figure 6. Delay time of each topology in 10 hours of simulation

- Real-time and historical alarms,

- Real-time and historical trends,

- Irrigation field,

- ZigBee network topology,

- Status of sensors deployed in the field, and

- Reports generation.

\subsection{Status of Sensors Deployed in the Field and ZigBee Network Topology}

In Figure 8 the designed topology is shown, and how it would be displayed in the field. There are a total of five final nodes that have soil temperature and humidity sensors. Due to the mesh topology, there are nodes that have 2 final devices, which in turn are connected to a router to be able to link to the coordinator node (central). Each sensor has configured alarm limit values, both higher and lower, and when any of these is exceeded, an alarm is generated in the system, an email is sent to the user and the element where it was generated is surrounded with a framework of color. The color of the frame depends on the severity of the alarm, for example, the red color indicates that it is an alarm number 1 and is urgent type. The alarm will be deactivated when the user recognizes the alarm, or when the value of the variable returns to a normal state.

\subsection{Irrigation Field}

In Figure 9 we have the crop scheme, along with the pipes and valves and the tanks that contain water and nutrients. The system monitors soil temperature and humidity values for each node. For example, if in a node, the temperature is high and the humidity in the ground is low, the system sends the order to activate the valve that sends water and nutrients to the area of the crop where the node is. In the opposite case, if the humidity is high the system sends the order to close the valve and thus only use the water and nutrients necessary in that area of the crop. In the same way, it is shown if the ZigBee device is active, or on the contrary it is inactive and therefore an alarm is generated.

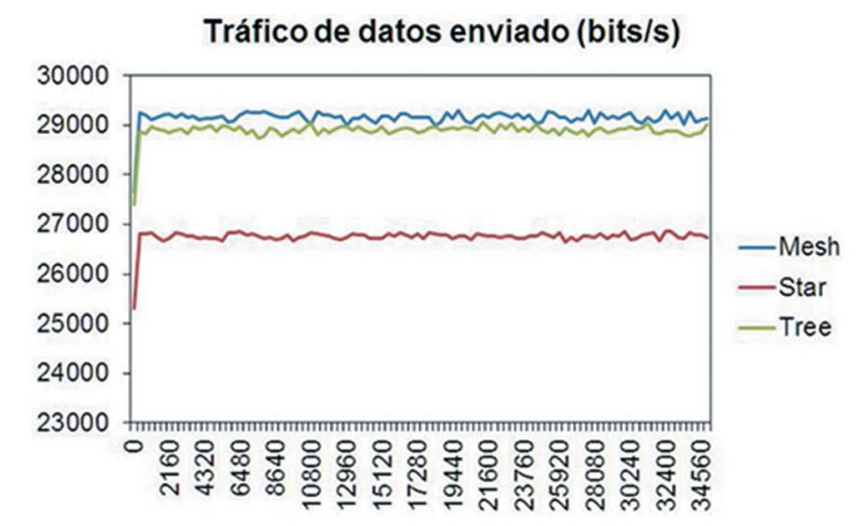

Figure 7. Data rate sent for each topology in 10 hours of simulation.

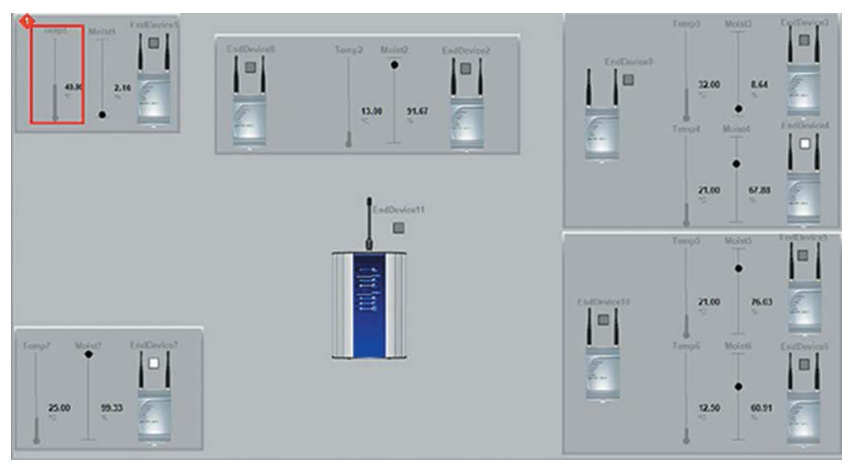

Figure 8. Level of the sensors and the current status of the final ZigBee device. 


\subsection{Real-time and historical trends}

In Figure 10 we can see the trend graph in real or historical time. The variables of temperature and humidity of the irrigation system are configured to store data, which are stored in plain text files, which can be consulted as many times as necessary. For example, information can be retrieved for 5 minutes, up to 2 months or more. The trend graph has a button to pause the graph in case of a detailed analysis of the information. If you want to see the information of a particular variable, there is a button that allows you to do this.

\subsection{Real-time and Historical Alarms}

Figure 11 show the alarm window, which is divided into two elements: the upper element consists of the table of alarms that are generated at run time and are not stored in any file. This table has 5 selectors on the left side, to display alarms according to the node where they were generated. In the lower part is the table of historical alarms, which can be stored, either in a plain text file, or in a table in database.

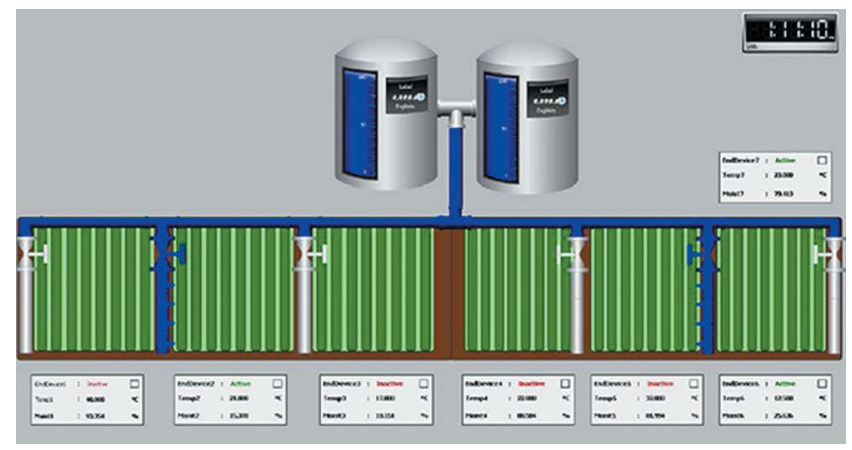

Figure 9. Cultivation together with two reservoirs (tanks) and the status of each node deployed

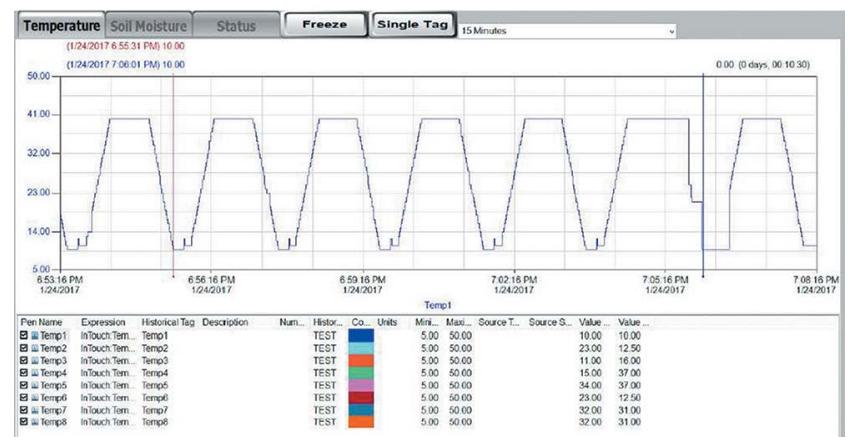

Figure 10. Graph of trends in real time and historical for each variable monitored.

\subsection{Reports Generation}

Figure 12 shows the window for generating manual reports. These can be generated manually, or automatically, by means of a command that is executed every certain period of time. For example, if a daily report of crop status is required, the command is scheduled to run once a day, and thus generate the document. In the case of manual reports, the user selects the duration of the report, whether in hours, days or weeks. Then select the period between data, that is, how often you want to see data in the report (every minute, every hour, etc.). You must also enter the date and time the report starts. The directory path where the SCADA application is hosted, the path where the historical files are located,

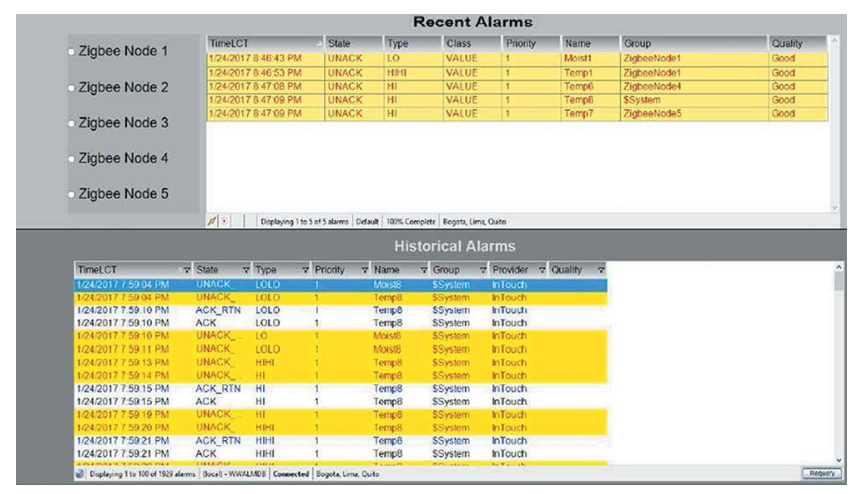

Figure 11. Alarms generated in real time (upper table) and those stored in database (lower table).

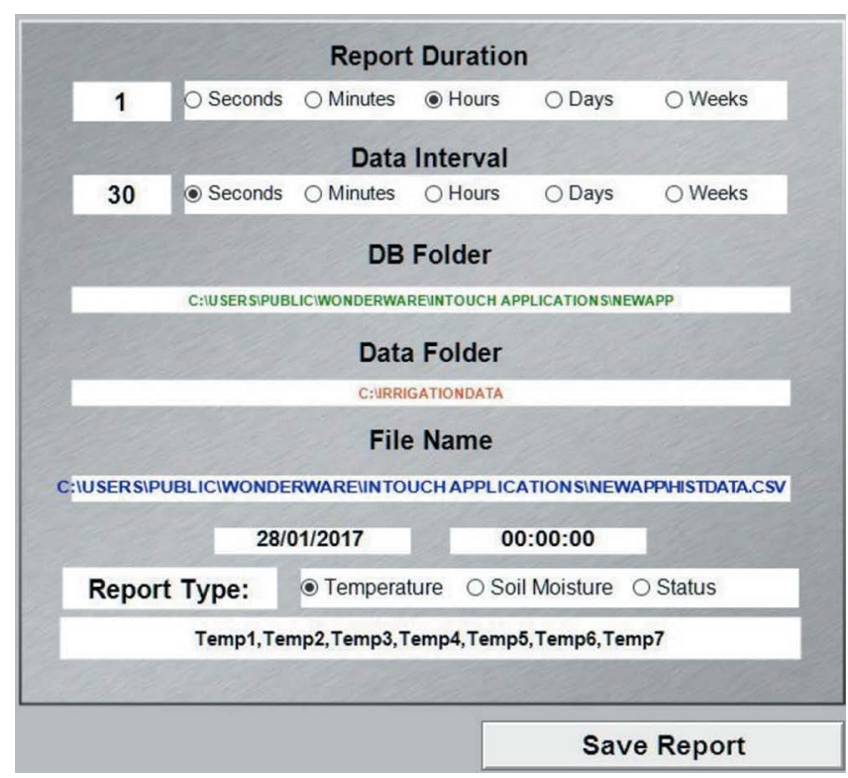

Figure 12. Generation of customized reports on crop variables 
and the path where the report is saved are also displayed. Depending on the type of report, the user can select temperature, humidity or status.

\section{Conclusions}

The work developed shows a detailed analysis to select from the irrigation method, the communication protocol and the sensors to be deployed. Since it is a design, it was made with 10 ZigBee devices, distributed in 5 nodes. The objective is that this serves as a basis for making applications on a larger scale.

The simulation of data acquisition from the crop was carried out to show the importance of storing the information of an agricultural process. It is also evident that the fact of involving new technologies in the automation of processes does not mean that they are expensive to implement, since as it was observed, it is not necessary to use databases to store the information, but using some system functionalities. SCADA could be stored in flat files, thus avoiding the purchase of a database manager's license, an aspect that would have increased the cost of the system.

The use of ZigBee devices as field devices, allows the realization of irrigation systems at low cost, since as described in section 4, the advantages it offers over the other protocols, in terms of costs and energy consumption are outstanding.

\section{References}

1. Lozano-EspitiaI, Ramírez-Villegas LM. How Productive is Rural Infrastructure? Evidence on Some Agricultural Crops in Colombia, Borradores de Economia. 2016; 948:1-22.

2. TECHNOSERVE. Construcción de un modelo deagriculturacompetitivaen Colombia. Bogotá D.C, Colombia; 2015. p. 1-123.

3. Chandra DG, Malaya DB. Role of e-Agriculture in Rural Development in Indian Context. International Conference on Emerging Trends in Networks and Computer Communications (ETNCC); 2011. p. 320-23.

4. Kobayashi K, Kobayashi F, Saito Y. Development of Agricultural Monitoring Application as Media for Social Interaction. SICE Annual Conference; 2011. p. 2065-68.

5. Urbano-Molano FA. Redes de Sensores Inalámbricos Aplicadas a Optimizaciónen Agriculturade Precisión para Cultivos de Café en Colombia, Chemical Engineering Science. 2012; 5(1):46-52.
6. He WQ, Cai MK, Wang YB, Wang XJ. Automatic water supply control system of graded constant pressure by variable frequency speed and its application to pipeline irrigation, Second WRI Global Congress on Intelligent Systems. 2010; 1:385-88. https://doi.org/10.1109/GCIS.2010.81.

7. Agrawal N, Singhal S. Smart drip irrigation system using raspberry pi and Arduino. In: International Conference on Computing, Communication and Automation, ICCCA; 2015. p. 928-32.

8. Kim Y, Evans RG. Software design for wireless sensor-based site-specific irrigation, Computers and Electronics in Agriculture. 2010; 66(2):159-65. https://doi.org/10.1016/j.compag.2009.01.007.

9. Díaz SE, Pérez JC, Mateos AC, Marinescu MC, Guerra BB. A novel methodology for the monitoring of the agricultural production process based on wireless sensor networks, Computers and Electronics in Agriculture. 2011; 79(2):252-65. https://doi.org/10.1016/j.compag.2011.02.004.

10. Joint Research Centre (JRC) of the European Commission. Precision Agriculture: An Opportunity for Eu Farmers- Potential Support with the Cap 2014-2020. Directorate-General for Internal Policies Policy Department B: Structural and Cohesion Policies; 2014. p. 1-56.

11. Gurban EH, Andreescu GD. SCADA element solutions using Ethernet and mobile phone network. IEEE 9th International Symposium on Intelligent Systems and Informatics; 2011. p. 303-08.

12. López-Riquelme JA, Soto F, Suardíaz J, Sánchez $P$, Iborra A, Vera JA. Wireless Sensor Networks for precision horticulture in Southern Spain, Computers and Electronics in Agriculture. 2009; 68(1):25-35. https://doi.org/10.1016/j.compag.2009.04.006.

13. Navarro-Hellín H, Martínez-del-Rincon J, DomingoMiguel R, Soto-Valles F, Torres-Sánchez. A decision support system for managing irrigation in agricultural, Computers and Electronics in Agriculture. 2016;124:121-31. https://doi.org/10.1016/j.compag.2016.04.003.

14. Capraro F, Pati-o D, Tosetti S, Schugurensky C. Neural network-based irrigation control for precision agriculture. IEEE International Conference on Networking, Sensing and Control; 2008. p. 357-62.

15. What are the disadvantages of Arduino? - Quora. Date accessed: 08/10/2014. https://www.quora.com/What-arethe-disadvantages-of-Arduino.

16. Raspberry Pi: What are its limitations? Date accessed: 25/04/2013. https://www.itproportal.com/2013/04/25/ raspberry-pi-what-are-its-limitations/.

17. Sohraby K, Minoli D, Znati T. Wireless Sensor Networks: Technology, Protocols, and Applications. John Wiley \& Sons, Inc.; 2006. p. 1-326. 
18. Patel N, Kathiriya H, Bavarva A. Wireless sensor network using ZigBee, International Journal of Research in Engineering and Technology. 2013; 2(6):1038-42. https://doi.org/10.15623/ijret.2013.0206021.

19. Introducción a Wi-Fi (802.11 o Wi-Fi). Date accessed: 16/10/2008. https://ccm.net/contents/802-introduction-towi-fi-802-11-or-wifi.

20. Krco S. Bluetooth Based Wireless Sensor Networks-Implementation Issues and Solutions. Invited Paper; 2002. p. 1-7.
21. Wang $N$, Zhang $N$, Wang $M$. Wireless sensors in agriculture and food industry - Recent development and future perspective, Computers and Electronics in Agriculture. 2006; 50(1):1-14. https://doi.org/10.1016/j.compag.2005.09.003.

22. Sutana SR, Krishan PB, Gouthami K, Kim TH. Wireless sensor network based remote environmental conditions monitoring system with GPRS, International Journal of Control and Automation. 2015; 8(10):73-80. https://doi.org/10.14257/ijca.2015.8.10.07. 